\title{
Structure of the gut microbiome following colonization with human feces determines colonic tumor burden
}

\author{
Nielson T Baxter ${ }^{1 \dagger}$, Joseph P Zackular ${ }^{1 \dagger}$, Grace Y Chen ${ }^{2}$ and Patrick D Schloss ${ }^{1 *}$
}

\begin{abstract}
Background: A growing body of evidence indicates that the gut microbiome plays a role in the development of colorectal cancer (CRC). Patients with CRC harbor gut microbiomes that are structurally distinct from those of healthy individuals; however, without the ability to track individuals during disease progression, it has not been possible to observe changes in the microbiome over the course of tumorigenesis. Mouse models have demonstrated that these changes can further promote colonic tumorigenesis. However, these models have relied upon mouse-adapted bacterial populations and so it remains unclear which human-adapted bacterial populations are responsible for modulating tumorigenesis.

Results: We transplanted fecal microbiota from three CRC patients and three healthy individuals into germ-free mice, resulting in six structurally distinct microbial communities. Subjecting these mice to a chemically induced model of CRC resulted in different levels of tumorigenesis between mice. Differences in the number of tumors were strongly associated with the baseline microbiome structure in mice, but not with the cancer status of the human donors. Partitioning of baseline communities into enterotypes by Dirichlet multinomial mixture modeling resulted in three enterotypes that corresponded with tumor burden. The taxa most strongly positively correlated with increased tumor burden were members of the Bacteroides, Parabacteroides, Alistipes, and Akkermansia, all of which are Gram-negative. Members of the Gram-positive Clostridiales, including multiple members of Clostridium Group XIVa, were strongly negatively correlated with tumors. Analysis of the inferred metagenome of each community revealed a negative correlation between tumor count and the potential for butyrate production, and a positive correlation between tumor count and the capacity for host glycan degradation. Despite harboring distinct gut communities, all mice underwent conserved structural changes over the course of the model. The extent of these changes was also correlated with tumor incidence.

Conclusion: Our results suggest that the initial structure of the microbiome determines susceptibility to colonic tumorigenesis. There appear to be opposing roles for certain Gram-negative (Bacteroidales and Verrucomicrobia) and Gram-positive (Clostridiales) bacteria in tumor susceptibility. Thus, the impact of community structure is potentially mediated by the balance between protective, butyrate-producing populations and inflammatory, mucin-degrading populations.
\end{abstract}

Keywords: Colorectal cancer, Community structure, Germ-free, Gut microbiome, Humanized mice, Microbiota

\footnotetext{
*Correspondence: pschloss@umich.edu

'Equal contributors

'Department of Microbiology and Immunology, University of Michigan, Ann

Arbor, Michigan, USA

Full list of author information is available at the end of the article
}

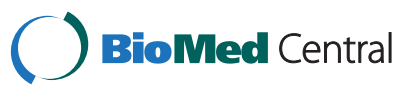

(c) 2014 Baxter et al.; licensee BioMed Central Ltd. This is an Open Access article distributed under the terms of the Creative Commons Attribution License (http://creativecommons.org/licenses/by/4.0), which permits unrestricted use, distribution, and reproduction in any medium, provided the original work is properly credited. The Creative Commons Public Domain Dedication waiver (http://creativecommons.org/publicdomain/zero/1.0/) applies to the data made available in this article, unless otherwise stated. 


\section{Background}

Colorectal cancer (CRC) is the second leading cause of cancer-related death in the United States each year [1]. Recent evidence suggests that the community of microbes inhabiting the gastrointestinal tract plays an important role in the development and progression of CRC [2-4]. This community, termed the gut microbiome, is known to influence cancer-related functions, including cell proliferation, angiogenesis, and apoptosis, and it is strongly linked to diet, obesity, and inflammation, which are known risk factors of CRC [5-9]. Using a mouse model of CRC, we have shown that structural changes to the microbiome occur during tumorigenesis and result in a gut microbiome with an increased tumorigenic capacity [10]. These findings demonstrate that the gut microbiome has a causal role in the development and progression of CRC.

Several survey-based studies have shown that CRC patients harbor microbial communities that are structurally distinct from those of healthy individuals [11-15]. However, there has been no consensus among these studies as to which bacterial populations are important. In mouse models, several gut commensals have been shown to promote tumorigenesis in the colon. Both enterotoxigenic Bacteroides fragilis (ETBF) and strains of Escherichia coli that carry the $p k s$ pathogenicity island can promote tumorigenesis by the production of toxins [3,4]. Fusobacterium nucleatum has also been shown to potentiate tumorigenesis in mouse models and cell culture experiments by stimulating inflammation via myeloid cell recruitment or activation of $\beta$-catenin signaling $[2,16]$. Fusobacterium was also found to be enriched in a subset of human colon adenomas [15]. Although there is increasing evidence that Fusobacterium is involved in CRC cases, it was detected in less than half of adenomas, which suggests that other bacterial populations are capable of potentiating tumorigenesis [2]. In fact, it may be that CRC is a polymicrobial disease requiring combinations of these or other populations to influence tumorigenesis.

While individual bacterial species have been associated with some human CRC cases, in other cases the capacity of the microbiome to modulate tumorigenesis could be determined by the structure of the community as a whole rather than the presence or absence of individual populations $[4,17]$. The potentially polymicrobial influence of the gut microbiome on this disease necessitates the disentangling of the complex interactions between bacterial populations in the gut. Understanding these interactions requires investigation of the relationship between the microbiome and tumorigenesis under a diverse set of community structures. Unfortunately, mechanistic studies typically rely on experiments with conventionally reared inbred mice living in homogenous, controlled environments, leading to relatively little variation in microbiome structure between individual animals. Although, experiments in con ventional mice are useful for understanding the mechanisms by which the microbiome modulates tumorigenesis, they are limited by investigating only those bacterial strains found in laboratory mice, many of which are absent in human beings. It is reasonable to expect that incorporating human-associated microbial populations into these experiments would increase the ability to translate results to human beings.

To investigate the role of microbiome structure in tumorigenesis, we combined the advantages of the high interpersonal variation among human beings and the convenience of a mouse model. We inoculated germ-free mice with microbiota from human subjects harboring distinct microbiomes. This technique enabled us to test the effect of different baseline microbiome communities with variation beyond what is seen in conventionally reared mice. The transfer of human microbiota to germ-free mice, sometimes referred to as 'humanization', has been employed to study the microbiome in the context of several other diseases. In studies of diabetes, obesity, and malnutrition, colonization with human feces has been reported to recapitulate the phenotype of the human donors in the recipient mice [18-21]. Thus, in addition to searching for tumor-modulating community structures, we sought to determine whether this strategy could be used to recapitulate the tumor-promoting capacity of CRC patients' microbiota in mice.

\section{Methods}

\section{Mouse experiments}

Fecal samples from three healthy individuals and three patients found to harbor carcinomas were obtained through the Early Detection Research Network (Additional file 1: Table S1). Diagnoses were determined based on colonoscopy and histology. All six samples were PCR-negative for the ETBF toxin and the E. coli pks island [4,22]. Collection of the human feces used in this study was approved by the University of Michigan Institutional Review Board. All enrollees granted consent to participate in the study. Inocula were prepared by mixing $200 \mathrm{mg}$ of each sample in 5 $\mathrm{ml}$ of PBS. Age-matched (6 to 10 weeks), male, germ-free C57BL/6 mice were inoculated by oral gavage with $100 \mu \mathrm{l}$ of inoculum ( $n=10$ for groups $\mathrm{H} 1$ and $\mathrm{C} 1, n=5$ for others). Mice were housed five mice per cage. Three weeks after inoculation, mice received a single intraperitoneal injection of azoxymethane (AOM; $10 \mathrm{mg} / \mathrm{kg}$ of body weight). Five days later, mice were subjected to the first of three five-day rounds of $2 \%$ dextran sulfate sodium (DSS) administered ad libitum in the drinking water (Figure 1). Sixteen days of recovery separated each round of DSS. Three weeks after the third and final round of DSS, mice were euthanized and colonic tumors were enumerated. With this model, mice consistently develop noninvasive adenomas with dysplastic changes [23,24]. Throughout 


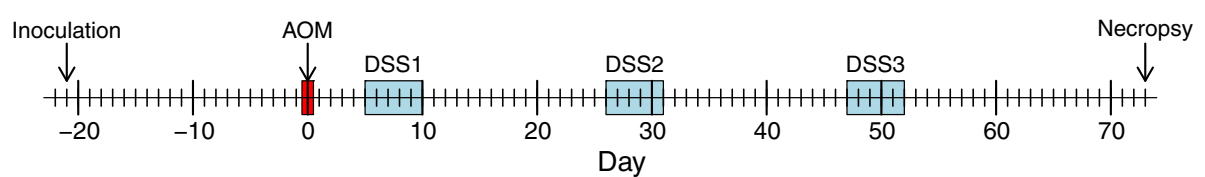

Figure 1 Experimental design. Germ-free mice were inoculated by oral gavage with one of six human inocula. Twenty-one days later (day 0), they received a single intraperitoneal injection of AOM (10 mg/kg). Mice were subsequently administered three five-day rounds of $2 \%$ DSS in the drinking water, with 16 days of rest in between. Mice were euthanized 73 days after the AOM injection for enumeration of colonic tumors. The inocula and samples collected on day 0 and day 73 were used for 165 rRNA gene sequencing. AOM, azoxymethane; DSS, dextran sulfate sodium.

the experiment, the mice were housed in germ-free isolators at the University of Michigan Germ-free Facility. This animal experiment was approved by the University Committee on Use and Care of Animals at the University of Michigan.

\section{DNA extraction and 16S rRNA gene sequencing}

Mouse fecal samples were collected throughout the experiment and frozen at $-20^{\circ} \mathrm{C}$. Genomic DNA from samples collected on days 0 and 73 and the human inocula were isolated using the PowerSoil-htp 96 Well Soil DNA isolation kit (MO BIO, Carlsbad, CA, USA) using an epMotion 5075 automated pipetting system. The V4 region of the $16 \mathrm{~S}$ rRNA gene was amplified using custom barcoded primers and sequenced as described previously using an Illumina MiSeq sequencer [25]. All fastq files and the MIMARKS spreadsheet are available online [26].

\section{Sequence curation and analysis}

The 16S rRNA gene sequences were curated using the mothur software package, as described previously [25,27]. Briefly, paired end reads were assembled into contigs and aligned to the SILVA 16S rRNA sequence database [28]. Sequences that failed to align or were flagged as possible chimeras by UCHIME were removed [29]. Each sequence was classified using a naive Bayesian classifier trained against a $16 \mathrm{~S}$ rRNA gene training set provided by the Ribosomal Database Project [30,31]. Finally, sequences were grouped based on their taxonomic classification or clustered into operational taxonomic units (OTUs) based on a $97 \%$ similarity cutoff. The number of sequences in each sample was rarefied to 3,306 sequences per sample, to minimize the effects of uneven sampling. Parallel sequencing and processing of a mock community indicated that the error rate of the curated sequences was $0.085 \%$.

The dissimilarity in community structure between samples was calculated using the $\Theta_{\mathrm{YC}}$ metric [32]. The $\Theta_{\mathrm{YC}}$ distances between samples were used for ordination analysis by nonmetric dimensional scaling (NMDS) in two dimensions. Ten iterations were performed and the resulting ordination that had the lowest stress was used for data visualization. Dirichlet multinomial mixture models were generated to group samples into enterotypes based on the abundance of bacterial genera in each sample [33]. To identify conserved changes that occurred over the course of the AOM/DSS model, the samples from each mouse on day 0 , and the samples collected at the end of the model were grouped into 'baseline' and 'endpoint' categories, respectively. The $\mathrm{R}$ randomForest package was used to identify the OTUs that best distinguished between the two categories based on their importance for the classification model $[34,35]$.

The Phylogenetic Investigation of Communities by Reconstruction of Unobserved States (PICRUSt) software package was used to infer the metagenomic content of each sample, based on the taxonomy and abundance of each OTU [36]. Although this method is limited by the number of available genomes, it has been shown to replicate metagenomes to a high degree of accuracy, especially for human-adapted bacterial communities. The weighted nearest sequenced taxon index (NSTI) for our samples was $0.056 \pm 0.01$. In general, NSTI values below 0.06 suggest that closely related reference genomes were available for the dataset [37]. From the inferred metagenomes, we identified KEGG orthologs that could be used as markers for butyrate production or host glycan degradation. Because either butyrate kinase or butyryl-CoA:acetate CoA-transferase is required for butyrate production in the gut, the KEGG orthologs chosen as markers for butyrate production were K00929 (butyrate kinase (EC:2.7.2.7)), K01034 (acetate CoAtransferase $\alpha$ subunit (EC:2.8.3.8)), K01035 (acetate CoAtransferase $\beta$ subunit (EC:2.8.3.8)) [38]. To choose markers for glycan degradation, we found all of the KEGG orthologs annotated as sialidases, fucosidases, sulfatases, or members of the glycoside hydrolase family 18 , as these classes of enzymes are necessary, and moderately specific for host glycan degradation [39,40]. Ten such KEGG orthologs were found in the metagenomes and used as markers: K01138 (uncharacterized sulfatase (EC:3.1.6.-)), K01130 (arylsufatase (EC:3.1.6.1)), K01135 (arylsufatase B (EC:3.1.6.12)), K01137 (N-acetylglucosamine-6-sulfatase (EC:3.1.6.14)), K01134 (arylsufatase A (EC:3.1.6.8)), K011 86 (sialidase-1 (EC:3.2.1.18)), K01206 ( $\alpha$-L-fucosidase (EC:3.2.151)), K01183 (1,4- $\beta$-poly- $N$-acetylglucosaminidase (EC:3.2.1.14)), K01205 ( $\alpha$ - $N$-acetylglucosaminidase (EC:3.2.1.50)), and K05970 (sialate O-acetylesterase 
(EC:3.1.1.53)). Finally, we calculated the Spearman correlation coefficients between tumor counts and these KEGG orthologs.

\section{Statistical analysis}

Differences in tumor counts between Dirichlet multinomial mixture (DMM) partitions were examined using a Wilcoxon rank-sum test. To test whether there was a significant difference in tumor counts between groups that received healthy or cancer-associated inocula, we rank transformed the tumor counts to correct for heteroscedasticity and performed a nested analysis of variance (ANOVA). Differences in community structure were examined using analysis of molecular variance (AMOVA) in mothur [41].

\section{Results}

\section{Colonization of germ-free mice with human microbiota}

We colonized germ-free mice with human feces from six individuals to determine whether different initial community structures would yield different numbers of tumors after going through the AOM/DSS model. This model was selected because the progression tumors in the AOM/DSS model closely resembles that of human CRC, including early mutations in APC or $\beta$-catenin signaling [42]. Furthermore, the model achieves colonic tumors and complete penetrance in the widely available C57BL/6 strain within 73 days. Three of the donors had healthy colons $(\mathrm{H} 1, \mathrm{H} 2, \mathrm{H} 3)$ and three had colonic carcinomas $(\mathrm{C} 1$, C2, C3). Samples were chosen because they represented broad variation in community structure (Figure 2A). Following gavage and a 21-day colonization period, groups showed varying levels of similarity to their inocula based on phylum level relative abundances and the $\Theta_{\mathrm{YC}}$ distances calculated from OTU abundances (Figure 2A,B). Low $\Theta_{Y C}$ distances between mice within groups suggested that individual communities were consistent within each group, while large $\Theta_{\mathrm{YC}}$ distances between groups suggested that each group harbored a gut microbiome that was structurally distinct from the others. Pairwise AMOVA between groups revealed that colonization with different inocula resulted in significantly different community structures $(P<0.01$, Benjamini-Hochberg correction). These results suggest that although mice do not closely resemble their inoculum, all sets of mice developed stable, structurally distinct gut communities.

Tumor incidence is linked to initial community structure Once colonized, mice were subjected to the AOM/DSS model of CRC. We observed significant variation in the number of tumors between mice (Figure 3A). These differences were associated with the inoculum they received, but not the cancer status of the human donor (nested ANOVA $P<0.0005)$. Thus, the phenotypes of the human subjects were not transferred to their mouse counterparts. Ordination of the communities revealed an association between the community structure of each group at the beginning of the AOM/DSS model and their median tumor counts (Figures $3 \mathrm{~B}$ and Additional file 1: Figure S1). To test for cage effects, groups $\mathrm{H} 1$ and $\mathrm{C} 1$ were each inoculated into duplicate cages of five mice each $(n=10$ per inoculum). There was no significant difference in baseline microbiome structure $(P>0.05$, AMOVA $)$ or tumor counts $(P>0.05$, Wilcoxon test) between cages within each group.

To determine which OTUs were driving this trend, we generated a biplot using the NMDS axes generated from the $\Theta_{\mathrm{YC}}$ distances between samples collected at the time of AOM injection (day 0; Figure 3B). Among the OTUs most strongly correlated with high tumor counts were two OTUs from the genus Bacteroides (OTUs 1 and 4). More detailed characterization of these OTUs indicated that OTU 1 was closely affiliated with $B$. uniformis and OTU 4 was affiliated with a mixture of Bacteroides species, including B. fragilis, B. ovatus, B. xylanisolvens, and B. thetaiotaomicron. Both of these OTUs were found in all six cohorts of mice and their initial abundances were positively correlated with tumor counts $(\rho=0.47$ and 0.49 , respectively; both $P<0.005$; Spearman correlation). Interestingly, all samples were PCR-negative for the ETBF toxin gene, suggesting that OTU 4 was not ETBF. Other OTUs associated with high tumor counts were affiliated with the genera Parabacteroides (OTU 18) and Alistipes (OTU 19), as well as an OTU affiliated with the species Akkermansia muciniphila (OTU 11). In addition, several OTUs associated with Clostridium Group XIVa (OTUs 7, 9, 15, and 17), Clostridium Group IV (OTU 49), and unclassified members of the Lachnospiraceae (OTUs 67 and 13) were correlated with lower tumor counts. These results indicate that the relative abundance of specific OTUs in the starting community could be associated with tumor counts.

To further test the association between the starting community structure and tumor incidence we clustered samples into community types using DMM models based on the abundance of bacterial genera found in the mice. This approach allowed us to quantify the association between the starting community structure and tumor burden in an unbiased manner. The DMM model with the highest likelihood partitioned the samples into three enterotypes (Figures 4A and Additional file 1: Figure S2). Enterotype 1 was composed exclusively of samples from the three treatment groups with the highest tumor counts (H2, C3, H1). Enterotype 2 was composed of samples from $\mathrm{C} 1$, which had the third lowest tumor count. Enterotype 3 was composed entirely of samples from the two groups with the lowest tumor counts (C2, H3). As a result, mice in enterotype 1 had significantly more 


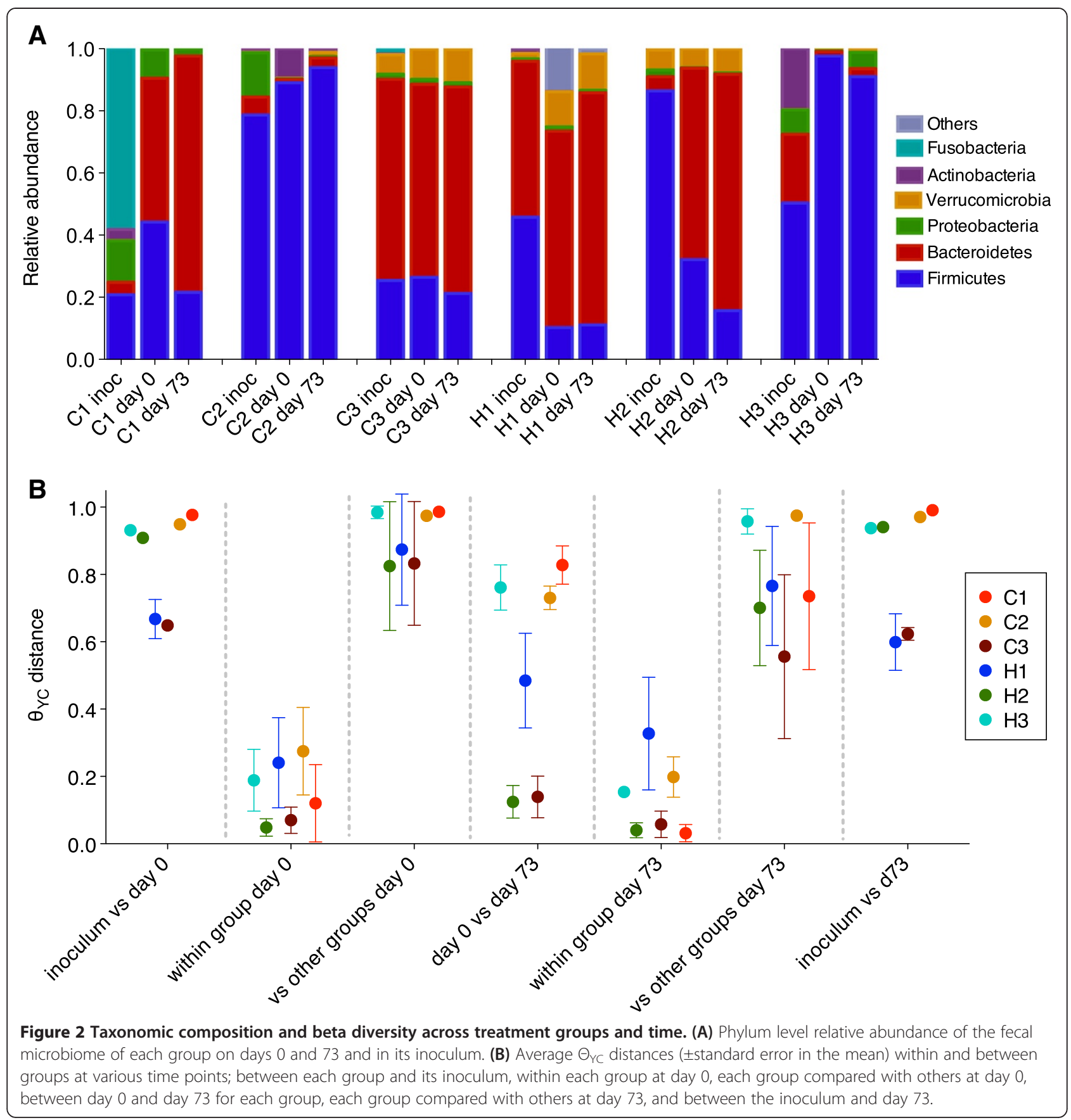

tumors than the other two partitions $(P<0.05$, Wilcoxon test; Figure $4 \mathrm{~B})$. Consistent with the OTU analysis, the DMM partition with the highest tumor counts was enriched for the genus Bacteroides (Figure 4C). In addition, other genera within the order Bacteroidales (Parabacteroides and Alistipes), as well as Akkermansia, were enriched in enterotype 1. An unclassified member of the Porphyromonodaceae, was enriched in enterotype 2, which had significantly fewer tumors than enterotype 1 . Enterotype 3 , which had the fewest tumors, was enriched for several genera within the order
Clostridiales (Clostridium Group XIVa, Clostridium Group XI, Clostridium Group XVIII, Flavonifractor, and unclassified Lachnospiraceae). These data suggest a potentially tumorigenic role for certain members of Bacteroidales and a protective role for certain members of Clostridiales.

\section{Changes in the microbiome during the AOM/DSS model}

To determine the extent to which the microbiomes of each group changed over the course of the AOM/DSS model, we calculated the $\Theta_{\mathrm{YC}}$ distances between the 

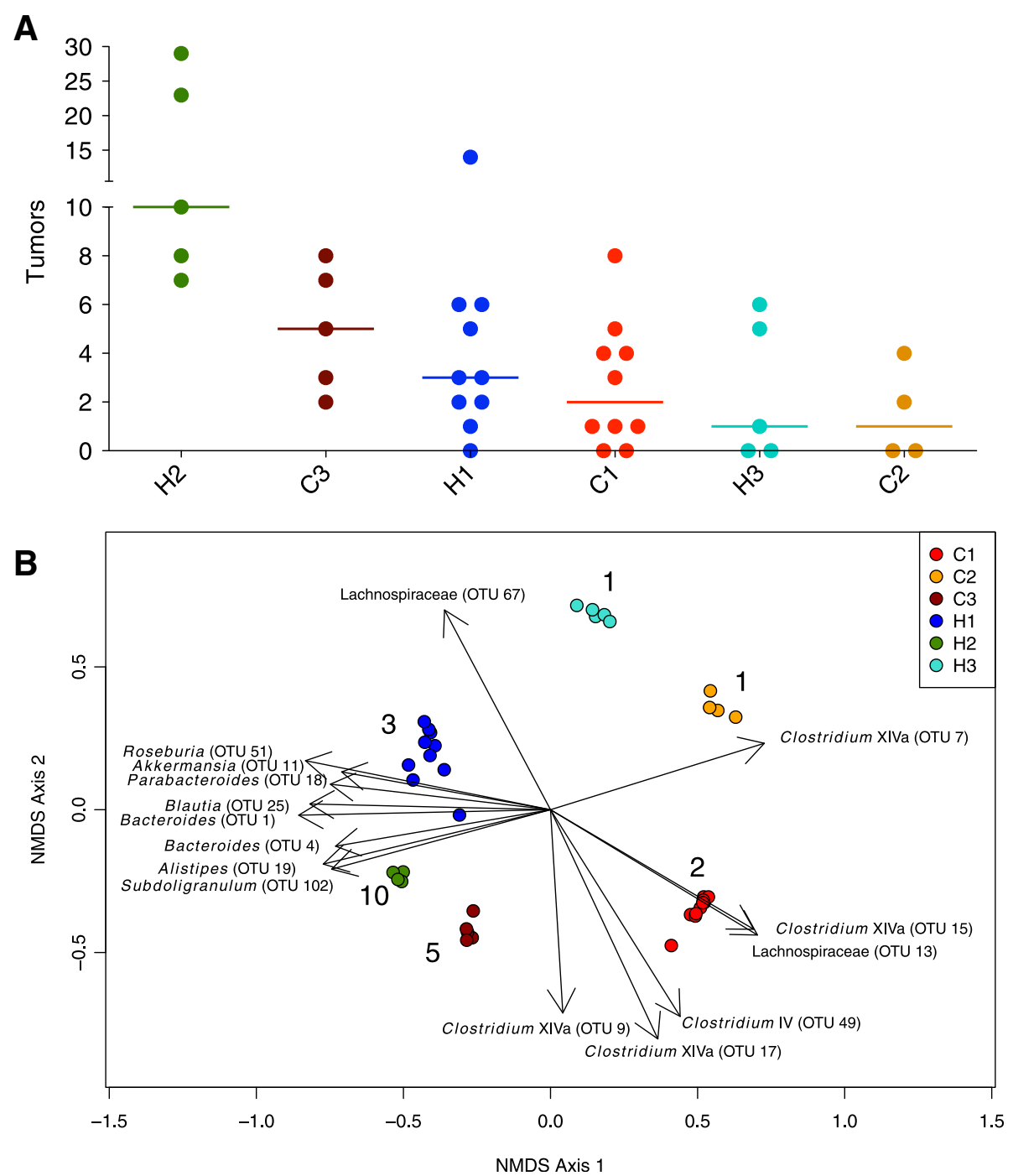

Figure 3 Correlation of tumor incidence with initial gut community structure. (A) Strip chart of tumor counts (with line at median) for each group. (B) NMDS plot based on $\Theta_{\text {YC }}$ distances between samples at day 0 with biplot of the 15 OTUs most strongly correlated with the NMDS axes (stress $=0.21$ ). Median tumor counts for each group are adjacent to their corresponding dots. NMDS, nonmetric dimensional scaling; OTU, operational taxonomic unit.

communities in mice at the time of AOM injection and at the end of the experiment. Interestingly, the two groups with the highest tumor counts $(\mathrm{H} 2, \mathrm{C} 3)$ changed very little over time $\left(\Theta_{Y C}=0.12\right.$ and 0.14$)$, while the microbiomes of the three groups with the lowest tumor counts $(C 2, H 3, C 1)$ changed substantially $\left(\Theta_{Y C}=0.73\right.$, $0.76,0.83$ ) (Figure $2 \mathrm{~A}$ ). Thus, the closer the initial community of each group was to the tumor-associated endpoint community, the more tumors those mice developed.

To identify which OTUs changed over time, we combined samples from all six treatment groups and used the Random Forest machine-learning algorithm to identify the OTUs that allowed us to differentiate between the samples from the beginning and end of the model, regardless of the inoculum. The resulting model was able to distinguish between the baseline and endpoint samples with $98.6 \%$ accuracy. The OTUs that provided the greatest mean decrease in accuracy when removed from the analysis were affiliated with Turicibacter (OTU 36), Bacteroides (OTU 4), Porphyromonadaceae (OTU 59), and several genera within the Clostridiales (OTUs 113, 25, 28, 127, 144, 42, and 17; Figure 5). Despite harboring drastically different community structures, all treatment groups underwent conserved changes in microbiome structure over the course of the model.

\section{Tumor incidence is linked to butyrate production and host glycan degradation}

Our experiments suggested that Clostridiales, Bacteroidales, and Akkermansia played a role in modulating tumorigenesis. 


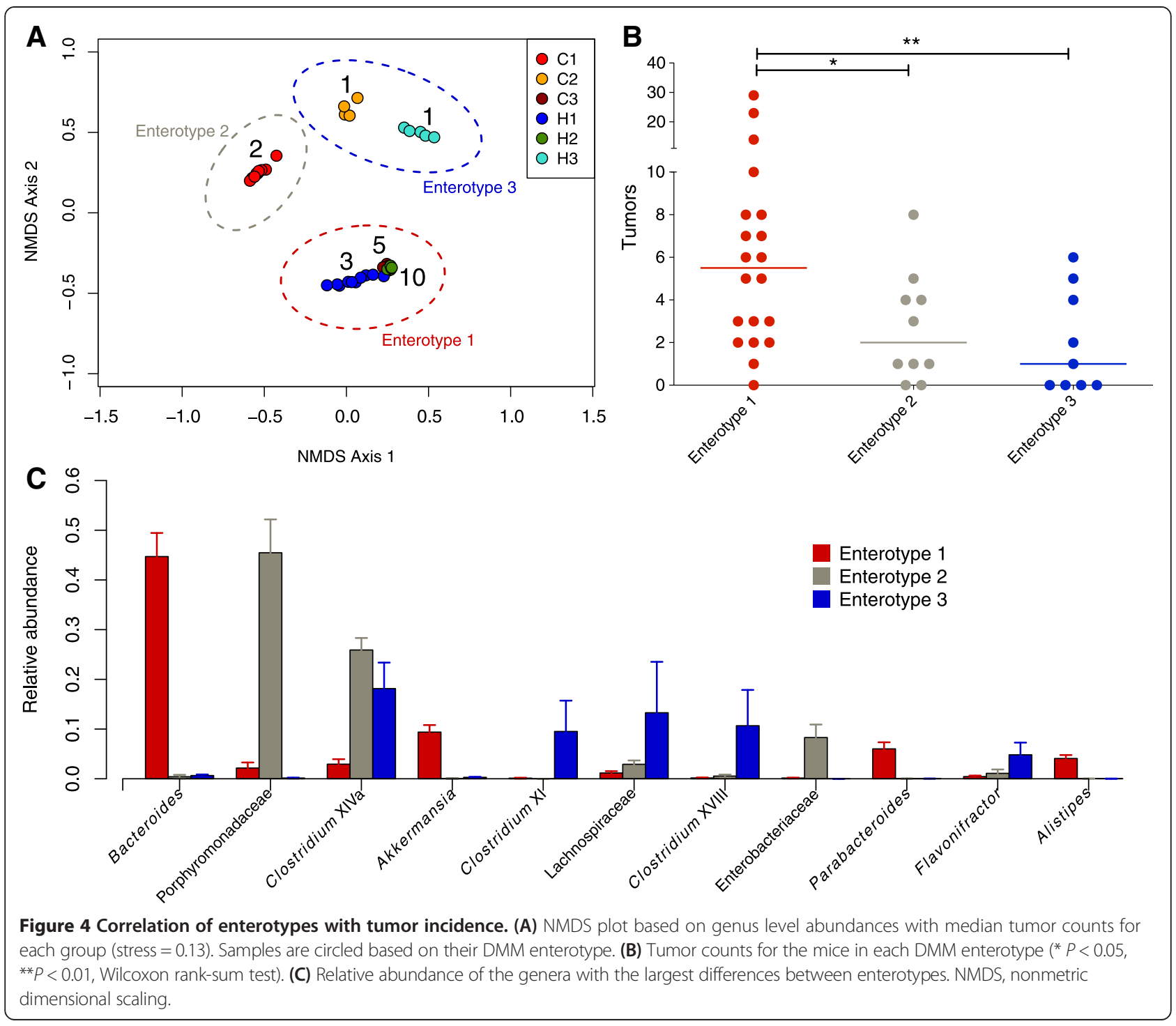

Members of the Clostridiales, especially Clostridium Group $\mathrm{XIVa}$, are the predominate producers of intestinal butyrate, an important anti-inflammatory and anti-tumorigenic metabolite in the gut $[38,43,44]$. Bacteroides and Akkermansia, on the other hand, are known to break down host-derived glycans, especially mucin. Mucin degradation has been linked to intestinal inflammation and can facilitate colonization of intestinal pathogens [45-47]. To test whether the genomic potential for these metabolic activities is linked to tumor incidence, we used the PICRUSt software package to predict the metagenomic content for each sample at the time of AOM injection. Butyrate production in the gut requires either butyryl-CoA:acetate CoA-transferase or butyrate kinase [32]. KEGG orthologs (KOs) of the $\alpha$ and $\beta$ subunits of butyryl-CoA:acetate CoA-transferase were negatively correlated with tumor incidence $(\rho<-0.35, P<$ $0.05)$. Butyrate kinase had the same trend, but the correlation was not statistically significant $(\rho=-0.30, P=0.08)$.
Next, we identified KOs for sialidases, fucosidases, sulfatases, and $N$-acetylglucosaminidases, which are indicative of host glycan degradation $[39,40]$. Of the ten such KOs found in our metagenomes, seven (two arylsulfatases, an uncharacterized sulfatase, $\alpha$-L-fucosidase, sialate $O$-acetylesterase, $\alpha-N$-acetylglucosaminidase, $1,4-\beta$-poly- $N$-acetylglucosaminidase) were positively correlated with tumor count $(\rho>0.47, P<0.01)$. None of the three remaining KOs correlated with tumors. Together, these data suggest that the correlation between tumor incidence and the microbiome might be dependent on metabolic activity rather than bacterial phylogeny.

\section{Discussion}

The results of this study demonstrate that the structure of the gut microbiome is important for determining susceptibility to inflammation-associated tumorigenesis. We observed strong correlations between the initial community 


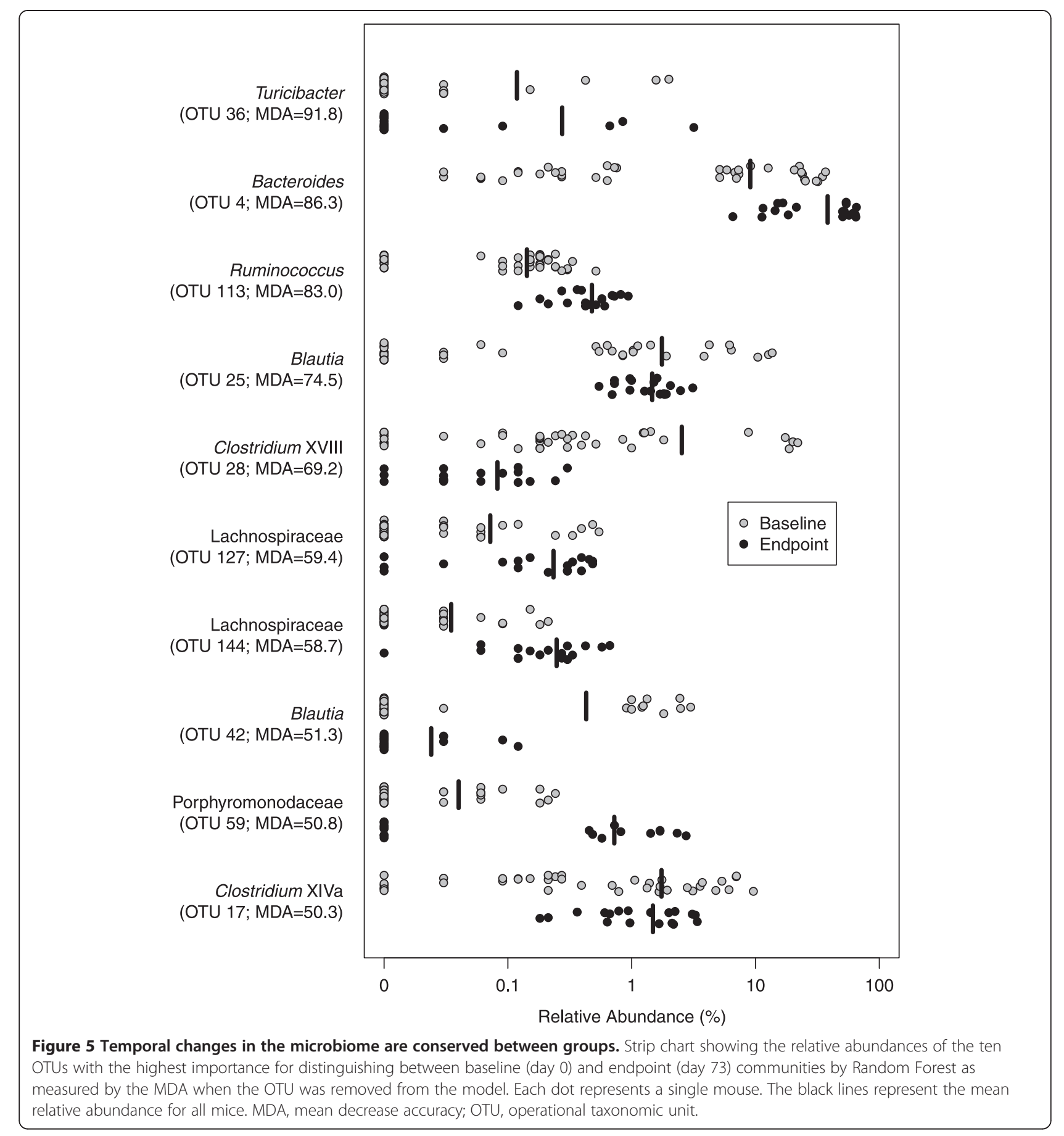

structure of the gut microbiome and tumor multiplicity. This relationship is driven primarily by two distinct groups of bacteria. In general, we found that members of the Bacteroidales (Bacteroides, Parabacteroides, Alistipes, and Porphyromonodaceae) were associated with a higher rate of tumorigenesis, while members of the Clostridiales, especially Clostridium Group XIVa, were associated with a decreased rate of tumorigenesis. There were exceptions to this pattern, however, as a few OTUs associated with
Clostridiales (OTUs associated with Roseburia, Blautia, and Subdoligranulum) were enriched in the groups with higher tumor counts (Figure 3B). However, these OTUs were less abundant $(<0.7 \%$ mean abundance) than those Clostridiales that were negatively correlated with tumors ( $\approx 2 \%$ mean abundance). Therefore, the data generally support a model in which susceptibility to colonic tumorigenesis is determined by the balance between the abundance of members of Bacteroidales and Clostridiales. One 
limitation of this study is that we only assayed the fecal communities. While this was necessary for correlating baseline community structure with the numbers of tumors that developed, characterization of the mucosal microbiota could potentially yield additional associations with tumor burden. It is also important to note that, although we observed variation in the number of tumors within inoculum groups, we were unable to correlate these differences with any differences in their microbiomes.

Based on our predicted metagenomic analysis, the roles of Clostridiales and Bacteroidales could be dependent on specific metabolic activities. Members of Clostridium Group XIVa are the predominant producers of butyrate in the gut [38]. Given the anti-inflammatory and antitumorigenic properties of intestinal butyrate, its production by members of Clostridium Group XIVa could explain the association with lower susceptibility to colon tumorigenesis $[43,44]$. This hypothesis is supported by our predicted metagenomic data, which correlated the increased potential for butyrate production with decreased tumorigenesis. Bacteroides and Akkermansia were the two genera most strongly correlated with higher rates of tumorigenesis. Both are known mucin degraders, and several genes linked to mucin degradation were positively correlated with tumor incidence. Additionally, previous studies have linked mucin degradation by Bacteroides and Akkermansia with intestinal inflammation [45-47]. It is possible that an overabundance of these or other mucin degraders could undermine the integrity of the mucosal barrier, leading to increased inflammation. Such a mechanism could be an alternative to the ETBF-based model of tumorigenesis, as we were unable to detect the gene for the ETBF toxin in any of our samples. While we cannot exclude the possibility of a novel toxin in the Bacteroides populations in our experiment, the additional correlation with Akkermansia muciniphila supports a model in which inflammation is induced by mucin degradation. If further experiments confirm this model, blocking mucin degradation could be used as a therapeutic for preventing or slowing the progression of tumorigenesis.

In this study, we observed a relationship between tumor multiplicity and the extent to which the microbiome shifted over the course of the model. The gut community of mice with high tumor counts changed very little over the course of the model, while the microbiome of groups with low tumor counts changed drastically. Thus, the more similar the baseline community was to the endpoint community, the more tumors the host developed. We hypothesize that the microbiome of these mice was not significantly altered by the AOM/DSS model since it was already in a state of dysbiosis. Therefore, there was a greater exposure to a tumorigenic microbiome. Similarly, in a previous study, we colonized germ-free mice with the feces of conventional mice that had already gone through the model [10]. These mice developed more tumors than germ-free mice colonized with feces from normal mice. Thus, in addition to needing a dysbiotic community to exacerbate tumorigenesis, the length of exposure to that community is important to tumor formation.

In contrast with earlier studies where human feces were used to colonize germ-free mice, we were unable to recapitulate the structures of the human microbiota donors, as numerous members of the donor community failed to colonize the recipients and others colonized in different abundances. For example, one of the donor communities (C1) was dominated by Fusobacterium species (58\% relative abundance). Another inoculum (C3), contained F. nucleatum at $2 \%$ relative abundance [2]. However, we did not recover any sequences from the phylum Fusobacteria in the recipient mice. We were also unable to culture it from the original human stool sample, suggesting that it might not have survived the freezing and thawing of the sample or was never alive in the stool. Colonizing germfree mice with human feces and recovering a similar microbiome is probably an unreasonable expectation. The mouse host certainly selects for specific populations of bacteria based on its immune system and metabolic profile $[48,49]$. In addition, some bacteria will only colonize after other bacteria have suitably prepared the environment. In the oral cavity, Fusobacterium only colonizes after streptococcal populations have first attached to the tooth surface [50]. Although we did not fully recapitulate the community structure or phenotype of the human donors, colonizing mice with human fecal communities did serve as a useful tool for generating novel community structures to test the influence of specific bacterial populations on tumorigenesis. This strategy also allowed us to investigate the role of human microbiota, which should be more clinically relevant, while maintaining the tractability of a mouse model.

\section{Conclusions}

In this study, we found that the process of colonizing germ-free mice with human fecal communities did not recapitulate the phenotype of the human donors in this particular mouse model of CRC. Nonetheless, our findings demonstrate the importance of the initial microbiome structure in determining the rate of tumorigenesis. Furthermore, we identified several bacterial populations correlated with tumor incidence in the context of six distinct gut communities. Multiple OTUs associated with the order Bacteroidales and the species Akkermansia muciniphila were correlated with exacerbated tumorigenesis, while several OTUs associated with Clostridium Group XIVa and other Clostridiales were correlated with protection. Based on inferred metagenomes of the baseline communities, we provided evidence that the positive correlations between Akkermansia and Bacteroidales and 
tumor incidence could be a result of their ability to degrade mucin, and the negative correlation between the Clostridiales and tumor incidence could be due to the production of butyrate. The results are consistent with a model in which susceptibility is determined by the balance between mucin degradation and short chain fatty acid production. More studies are needed to confirm these results and to test the mechanisms by which these or other bacterial populations influence colon tumorigenesis. A better understanding of microbiome structures with a propensity to promote or inhibit tumorigenesis could lead to the development of prebiotic or probiotic therapies to prevent or slow the development and progression of CRC.

\section{Additional file}

Additional file 1: Table S1. Metadata for the six inoculum donors. Figure S1. Temporal changes in community structure. NMDS ordination based the differences in OTU abundances between samples on day 0 and day 73. Distances were calculated with $\Theta_{Y c}$. Figure S2. Samples remain in same enterotypes over the course of the model. NMDS ordination showing DMM enterotypes generated based on genus level abundances on day 73. Distances were calculated with $\Theta_{Y C}$. Despite changes in OTU abundance over the course of the model, all mice clustered into the same enterotypes on day 73 as they did on day 0 .

\section{Abbreviations}

AMOVA: analysis of molecular variance; ANOVA: analysis of variance; AOM: azoxymethane; CRC: colorectal cancer; DMM: Dirichlet multinomial mixture; DSS: dextran sulfate sodium; ETBF: enterotoxigenic Bacteroides fragilis; KO: KEGG ortholog; NMDS: nonmetric dimensional scaling; NSTI: nearest sequenced taxon index; OTU: operational taxonomic unit; PBS: phosphate-buffered saline; PCR: polymerase chain reaction; PICRUSt: Phylogenetic Investigation of Communities by Reconstruction of Unobserved States.

\section{Competing interests}

The authors declare that they have no competing interests.

\section{Authors' contributions}

NTB and JPZ designed and carried out the study, performed the bioinformatic and statistical analysis, and wrote the manuscript. GYC and PDS helped design and write the manuscript. All authors read and approved the final manuscript.

\section{Acknowledgements}

This work was supported by grants from the National Institutes for Health to PDS (R01GM099514, R01HG005975, P30DK034933, and University of Michigan GI SPORE) and GYC (University of Michigan GI SPORE and ARRA Supplement P30CA4659-22S3). The funding agencies had no role in study design, data collection and analysis, decision to publish, or preparation of the manuscript.

\section{Author details}

'Department of Microbiology and Immunology, University of Michigan, Ann Arbor, Michigan, USA. ${ }^{2}$ Department of Internal Medicine, Division of Hematology and Oncology, University of Michigan, Ann Arbor, Michigan, USA.

Received: 5 March 2014 Accepted: 4 June 2014

Published: 17 June 2014

\section{References}

1. American Cancer Society: Colorectal Cancer Facts \& Figures 2011-2013. Atlanta: 2013.
2. Kostic AD, Chun E, Robertson L, Glickman JN, Gallini CA, Michaud M, Clancy TE, Chung DC, Lochhead P, Hold GL, El-Omar EM, Brenner D, Fuchs CS, Meyerson M, Garrett WS: Fusobacterium nucleatum potentiates intestinal tumorigenesis and modulates the tumor-immune microenvironment. Cell Host Microbe 2013, 14(2):207-215.

3. Wu S, Rhee KJ, Albesiano E, Rabizadeh S, Wu X, Yen HR, Huso DL, Brancati FL, Wick E, McAllister F, Housseau F, Pardoll DM, Sears CL: A human colonic commensal promotes colon tumorigenesis via activation of $\mathrm{T}$ helper type 17 T cell responses. Nat Med 2009, 15(9):1016-1022.

4. Arthur JC, Perez-Chanona E, Mühlbauer M, Tomkovich S, Uronis JM, Fan TJ, Campbell BJ, Abujamel T, Dogan B, Rogers AB, Rhodes JM, Stintzi A, Simpson KW, Hansen JJ, Keku TO, Fodor AA, Jobin C: Intestinal inflammation targets cancer-inducing activity of the microbiota. Science 2012, 338(6103):120-123.

5. Cheesman SE, Neal JT, Mittge E, Seredick BM, Guillemin K: Epithelial cell proliferation in the developing zebrafish intestine is regulated by the Wnt pathway and microbial signaling via Myd88. Proc Natl Acad Sci USA 2011, 108(Suppl 1):4570-4577.

6. Dolara P, Caderni G, Salvadori M, Morozzi G, Fabiani R, Cresci A, Orpianesi C, Trallori G, Russo A, Palli D: Fecal levels of short-chain fatty acids and bile acids as determinants of colonic mucosal cell proliferation in humans. Nutr Cancer 2002, 42(2):186-190.

7. Stappenbeck TS, Hooper LV, Gordon Jl: Developmental regulation of intestinal angiogenesis by indigenous microbes via Paneth cells. Proc Natl Acad Sci USA 2002, 99(24):15451-15455.

8. Huxley RR, Ansary-Moghaddam A, Clifton P, Czernichow S, Parr CL, Woodward $\mathrm{M}$ : The impact of dietary and lifestyle risk factors on risk of colorectal cancer: a quantitative overview of the epidemiological evidence. Int J Cancer 2009, 125(1):171-180.

9. Chambers WM, Warren BF, Jewell DP, Mortensen NJM: Cancer surveillance in ulcerative colitis. Br J Surg 2005, 92(8):928-936.

10. Zackular JP, Baxter NT, Iverson KD, Sadler WD, Petrosino JF, Chen GY, Schloss PD: The gut microbiome modulates colon tumorigenesis. mBio 2013, 4(6):e00692-e00613.

11. Wang T, Cai G, Qiu Y, Fei N, Zhang M, Pang X, Jia W, Cai S, Zhao L: Structural segregation of gut microbiota between colorectal cancer patients and healthy volunteers. ISME J 2012, 6(2):320-329.

12. Chen HM, Yu YN, Wang JL, Lin YW, Kong X, Yang CQ, Yang L, Liu ZJ, Yuan YZ, Liu F, Wu JX, Zhong L, Fang DC, Zou W, Fang JY: Decreased dietary fiber intake and structural alteration of gut microbiota in patients with advanced colorectal adenoma. Am J Clin Nutr 2013, 97(5):1044-1052.

13. Chen W, Liu F, Ling Z, Tong $X$, Xiang C: Human intestinal lumen and mucosa-associated microbiota in patients with colorectal cancer. PLoS One 2012, 7(6):e39743.

14. Shen XJ, Rawls JF, Randall T, Burcal L, Mpande CN, Jenkins N, Jovov B, Abdo Z, Sandler RS, Keku TO: Molecular characterization of mucosal adherent bacteria and associations with colorectal adenomas. Gut Microbes 2010, 1(3):138-147.

15. Kostic AD, Gevers D, Pedamallu CS, Michaud M, Duke F, Earl AM, Ojesina Al, Jung J, Bass AJ, Tabernero J, Baselga J, Liu C, Shivdasani RA, Ogino S, Birren BW, Huttenhower C, Garrett WS, Meyerson M: Genomic analysis identifies association of Fusobacterium with colorectal carcinoma. Genome Res 2012, 22(2):292-298.

16. Rubinstein MR, Wang X, Liu W, Hao Y, Cai G, Han YW: Fusobacterium nucleatum promotes colorectal carcinogenesis by modulating $\mathrm{E}$ cadherin/ß-catenin signaling via its FadA adhesin. Cell Host Microbe 2013, 14(2):195-206.

17. Toprak NU, Yagci A, Gulluoglu BM, Akin ML, Demirkalem P, Celenk T, Soyletir $\mathrm{G}$ : A possible role of Bacteroides fragilis enterotoxin in the aetiology of colorectal cancer. Clin Microbiol Infec 2006, 12(8):782-786.

18. Alpert C, Sczesny S, Gruhl B, Blaut M: Long-term stability of the human gut microbiota in two different rat strains. Curr Issues Mol Biol 2008, 10(1-2):17-24.

19. Turnbaugh PJ, Ley RE, Mahowald MA, Magrini V, Mardis ER, Gordon J: An obesity-associated gut microbiome with increased capacity for energy harvest. Nature 2006, 444(7122):1027-1031.

20. Koren O, Goodrich JK, Cullender TC, Spor A, Laitinen K, Bäckhed HK, Gonzalez A, Werner JJ, Angenent LT, Knight R, Bäckhed F, Isolauri E, Salminen S, Ley RE: Host remodeling of the gut microbiome and metabolic changes during pregnancy. Cell 2012, 150(3):470-480.

21. Smith MI, Yatsunenko T, Manary MJ, Trehan I, Mkakosya R, Cheng J, Kau AL, Rich SS, Concannon P, Mychaleckyj JC, Liu J, Houpt E, Li JV, Holmes E, 
Nicholson J, Knights D, Ursell LK, Knight R, Gordon Jl: Gut microbiomes of Malawian twin pairs discordant for kwashiorkor. Science 2013, 339(6119):548-554.

22. Franco AA, Cheng RK, Goodman A, Sears CL: Modulation of bft expression by the Bacteroides fragilis pathogenicity island and its flanking region. Mol Microbiol 2002, 45(4):1067-1077.

23. Chen GY, Shaw MH, Redondo G, Nunez G: The innate immune receptor Nod1 protects the intestine from inflammation-induced tumorigenesis. Cancer Res 2008, 68(24):10060-10067.

24. Tanaka T, Kohno H, Suzuki R, Yamada Y, Sugie S, Mori H: A novel inflammation-related mouse colon carcinogenesis model induced by azoxymethane and dextran sodium sulfate. Cancer Sci 2003, 94(11):965-973.

25. Kozich JJ, Westcott SL, Baxter NT, Highlander SK, Schloss PD: Development of a dual-index sequencing strategy and curation pipeline for analyzing amplicon sequence data on the MiSeq Illumina sequencing platform. Appl Environ Microbiol 2013, 79(17):5112-5120.

26. Schloss P: Data File Repository for the Manuscript, 'Structure of the Gut Microbiome Following Colonization with Human Feces Determines Colonic Tumor Burden' by Niel Baxter, Joseph Zackular and Colleagues. http://www.mothur.org/human mouse aomdss.

27. Schloss PD, Westcott SL, Ryabin T, Hall JR, Hartmann M, Hollister EB, Lesniewski RA, Oakley BB, Parks DH, Robinson CJ, Sahl JW, Stres B, Thallinger GG, Van Horn DJ, Weber CF: Introducing mothur: open-source, platformindependent, community-supported software for describing and comparing microbial communities. App/ Environ Microbiol 2009, 75(23):7537-7541.

28. Pruesse E, Quast C, Knittel K, Fuchs BM, Ludwig W, Peplies J, Glockner FO: SILVA: a comprehensive online resource for quality checked and aligned ribosomal RNA sequence data compatible with ARB. Nucleic Acids Res 2007, 35(21):7188-7196.

29. Edgar RC, Haas BJ, Clemente JC, Quince C, Knight R: UCHIME improves sensitivity and speed of chimera detection. Bioinformatics 2011, 27(16):2194-2200.

30. Wang Q, Garrity GM, Tiedje JM, Cole JR: Naive Bayesian classifier for rapid assignment of rRNA sequences into the new bacterial taxonomy. Appl Environ Microbiol 2007, 73(16):5261-5267.

31. RDP Classifier. http://sourceforge.net/projects/rdp-classifier/.

32. Yue JC, Clayton MK: A similarity measure based on species proportions. Commun Stat Theory Methods 2005, 34(11):2123-2131.

33. Holmes I, Harris K, Quince C: Dirichlet multinomial mixtures: generative models for microbial metagenomics. Plos One 2012, 7(2):e30126.

34. Breiman L: Random forests. Mach Learn 2001, 45(1):5-32.

35. Liaw A, Wiener M: Classification and regression by randomForest. $R$ News 2002, 2:18-22.

36. Langille MG, Zaneveld J, Caporaso JG, McDonald D, Knights D, Reyes JA, Clemente JC, Burkepile DE, Vega Thurber RL, Knight R, Beiko RG, Huttenhower C: Predictive functional profiling of microbial communities using 16S rRNA marker gene sequences. Nat Biotechnol 2013, 31(9):814-821.

37. Quality Control of PICRUST Predictions. http://picrust.github.io/picrust/ tutorials/quality_control.html.

38. Pryde SE, Duncan SH, Hold GL, Stewart CS, Flint HJ: The microbiology of butyrate formation in the human colon. FEMS Microbiol Lett 2002, 217(2):133-139.

39. Corfield AP, Wagner SA, Clamp JR, Kriaris MS, Hoskins LC: Mucin degradation in the human colon: production of sialidase, sialate $\mathrm{O}$-acetylesterase, $\mathrm{N}$-acetylneuraminate lyase, arylesterase, and glycosulfatase activities by strains of fecal bacteria. Infect Immun 1992 60(10):3971-3978.

40. Martens EC, Chiang HC, Gordon Jl: Mucosal glycan foraging enhances fitness and transmission of a saccharolytic human gut bacterial symbiont. Cell Host Microbe 2008, 4(5):447-457.

41. Excoffier L, Smouse PE, Quattro JM: Analysis of molecular variance inferred from metric distances among DNA haplotypes: application to human mitochondrial DNA restriction data. Genetics 1992, 131(2):479-491.

42. De Robertis M, Massi E, Poeta ML, Carotti S, Morini S, Cecchetelli L, Signori E, Fazio VM: The AOM/DSS murine model for the study of colon carcinogenesis: from pathways to diagnosis and therapy studies. J Carcinog 2011, 10:9.
43. Segain JP, Raingeard de la Bletiere D, Bourreille A, Leray V, Gervois N, Rosales C, Ferrier L, Bonnet C, Blottiere HM, Galmiche JP: Butyrate inhibits inflammatory responses through NFKB inhibition: implications for Crohn's disease. Gut 2000, 47(3):397-403.

44. Hague A, Elder DJ, Hicks DJ, Paraskeva C: Apoptosis in colorectal tumour cells: induction by the short chain fatty acids butyrate, propionate and acetate and by the bile salt deoxycholate. Int J Cancer 1995, 60(3):400-406.

45. Bloom SM, Bijanki VN, Nava GM, Sun L, Malvin NP, Donermeyer DL, Dunne WM, Allen PM, Stappenbeck TS: Commensal Bacteroides species induce colitis in host-genotype-specific fashion in a mouse model of inflammatory bowel disease. Cell Host Microbe 2011, 9(5):390-403.

46. Ng KM, Ferreyra JA, Higginbottom SK, Lynch JB, Kashyap PC, Gopinath S, Naidu N, Choudhury B, Weimer BC, Monack DM, Sonnenburg JL: Microbiota-liberated host sugars facilitate post-antibiotic expansion of enteric pathogens. Nature 2013, 502(7469):96-99.

47. Ganesh BP, Klopfleisch R, Loh G, Blaut M: Commensal Akkermansia muciniphila exacerbates gut inflammation in Salmonella Typhimuriuminfected gnotobiotic mice. Plos One 2013, 8(9):e74963.

48. Rawls JF, Mahowald MA, Ley RE, Gordon JI: Reciprocal gut microbiota transplants from zebrafish and mice to germ-free recipients reveal host habitat selection. Cell 2006, 127(2):423-433.

49. Chung H, Pamp SJ, Hill JA, Surana NK, Edelman SM, Troy EB, Reading NC, Villablanca EJ, Wang S, Mora JR, Umesaki Y, Mathis D, Benoist C, Relman DA, Kasper DL: Gut immune maturation depends on colonization with a host-specific microbiota. Cell 2012, 149(7):1578-1593.

50. Kolenbrander PE, Palmer RJ Jr, Periasamy S, Jakubovics NS: Oral multispecies biofilm development and the key role of cell-cell distance. Nat Rev Microbiol 2010, 8(7):471-480.

doi:10.1186/2049-2618-2-20

Cite this article as: Baxter et al:: Structure of the gut microbiome following colonization with human feces determines colonic tumor burden.

Microbiome 2014 2:20

\section{Submit your next manuscript to BioMed Central and take full advantage of:}

- Convenient online submission

- Thorough peer review

- No space constraints or color figure charges

- Immediate publication on acceptance

- Inclusion in PubMed, CAS, Scopus and Google Scholar

- Research which is freely available for redistribution 